\title{
Gesundheitspolitik aus der Optik der Schweizer Ärztinnen und Ärzte
}

«Wie sieht die Ärzteschaft die medizinische und gesundheitspolitische Entwicklung bis ins Jahr 2010? Welches realisierbare Modell einer zukunfts orientierten Gesundheitspolitik soll sie nach aussen kommunizieren?» Diese Grundfragen standen am Anfang der VEDAGKlausurtagung (Präsidenten und Mitglieder aus den Vorständen der im VEDAG vertretenen kantonalen Ärztegesellschaften) vom 1. und 2. November 2002 in Quarten. Nach einleitenden Referaten und Diskussionen mit Vertreterinnen und Vertretern verschiedener Player im Gesundheitswesen wurden die Grundsätze zu den vorliegenden
Thesen erarbeitet. Nach mehreren Vernehmlassungen innerhalb des VEDAG entstanden unter der Redaktion Dr. med. Andreas Haefelis (Co-Präsident VEDAG bis 18. März 2004) die vorliegenden Thesen in der Schlussfassung vom 13. April 2004. Sie sollen uns als Grundlage dienen für unseren Prozess der Positionierung gegenüber Patienten, Partnern im Gesundheitswesen und der Politik. In diesem Sinne sollen sie auch in das «Konzept Öffentlichkeitsarbeit FMH» einfliessen.

Dr. med. Christoph Ramstein, Co-Präsident VEDAG

Dieses Editorial von Christoph Ramstein hätte in der Nr. 29/30 der SÄZ erscheinen sollen. Es bezieht sich auf den dort publizierten Text des VEDAG «Gesundheitspolitik aus der Optik der Schweizer Ärztinnen und Ärzte» [Schweiz Ärztezeitung 2004;85(29/30):1531-4]. Die Redaktion entschuldigt sich für diesen Fehler. 


\section{La politique de la santé selon les médecins suisses}

«Comment le corps médical voit-il l'évolution de la médecine et la politique de la santé jusqu'en 2010? Quel modèle réalisable d'une politique de la santé axée sur l'avenir doit-il communiquer au public?» C'est par ces questions fondamentales que s'est ouverte la séance à huis-clos du VEDAG (présidents et membres des comités des sociétés cantonales de médecine représentées au VEDAG) des $1^{\text {er }}$ et 2 novembre 2002 à Quarten. Après des exposés introductifs et des discussions avec différents acteurs de la santé publique ou leurs représentants, la séance a porté sur l'élaboration des principes directeurs. Après plusieurs consultations au sein du VEDAG, les présentes thèses (version finale du 13 avril 2004) ont vu le jour à l'initiation du Dr Andreas Haefeli (coprésident du VEDAG jusqu'au 18 mars 2004). Elles constituent la base sur laquelle nous établirons nos positions face aux patients et aux partenaires de la santé publique et de la politique. Elles pourront aussi, à ce titre, être intégrées dans le «Concept de relations publiques de la $\mathrm{FMH}$ ».

Dr Christoph Ramstein, co-président du VEDAG

Cet éditorial de Christoph Ramstein aurait dû paraître au numéro 31 du BMS. Il se réfère au texte publié par le VEDAG (Verband deutschschweizerischer Ärztegesellschaften) «La politique de la santé selon les médecins suisses» [Bull méd suisses 2004;85(31):1602-5]. La rédaction demande de bien vouloir excuser cette omission. 